\title{
Cardiorespiratory Arrest Associated with Propranolol use in Thyrotoxic Heart Disease
}

\author{
Lam AHY* \\ School of Nursing, The University of Hong Kong, Hong Kong \\ *Corresponding author: Lam AHY, School of Nursing, The University of Hong Kong, 4/F, William MW Mong Block, 21 Sassoon Road, Pokfulam, Hong \\ Kong
}

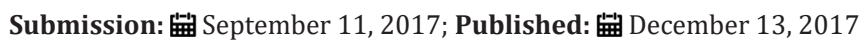

\section{Summary}

Beta-blockers are commonly used to treat tachyarrhythmia's in thyroid storm. However, there have been several cases in which cardiovascular collapse has been reported after administration of Propranolol [1]. This phenomenon seemed to be more common in Chinese [1]. The article presented an unusual case. A 14-year-old girl with thyrotoxicosis presented with wide complex tachycardia in an electrocardiogram (ECG). The rhythm was converted to a trial tachycardia after cardio version. Propranolol was started to control atrial tachycardia. The girl developed cardiorespiratory arrest soon after taking Propranolol. She required high-dose inotropic support and the use of an intra-aortic balloon pump after resuscitation.

Propranolol should be used highly cautiously in patients with thyrotoxic heart failure; cardiac assessment can be performed before medication prescription; short-acting beta-blockers may be a safer option for these patients [2].

\section{Background}

Although thyrotoxic patients presenting with tachyarrhythmia are conventionally treated with Propranolol to control heart rate and beta-receptor mediated symptoms such as anxiety and tremulousness [3], administration of Propranolol causes left ventricular ejection fraction (LVEF) to fall to a subnormal or even fatal level in patients with thyrotoxic cardiac failure [4]. The article presents a crucial clinical reminder of the general principles of conventional treatment by beta-blockers in thyrotoxic-induced tachyarrhythmia.

\section{Case Presentation}

A 14-year-old girl with good past health complained of palpitation for 4 months. On the date of admission, she complained of dizziness, shortness of breath, restlessness, and palpitation. She was a febrile. She remained conscious and her blood pressure (BP) was 108/58mmHg. Her electrocardiography (ECG) showed wide complex tachycardia with a rate of $>200 /$ min (Figure 1 ). The cardiac rhythm was not responsive to Adenosine and Amiodarone intravenous infusion. Doctors performed cardioversion 100 J (biphasic) and the rhythm was reverted to multifocal atrial tachycardia (Figure 2). An amiodarone maintenance oral dose of $600 \mathrm{mg}$ daily was started. Her ECG rhythm remained multifocal atrial tachycardia with non-sustain edventricular tachycardia (NSVT).

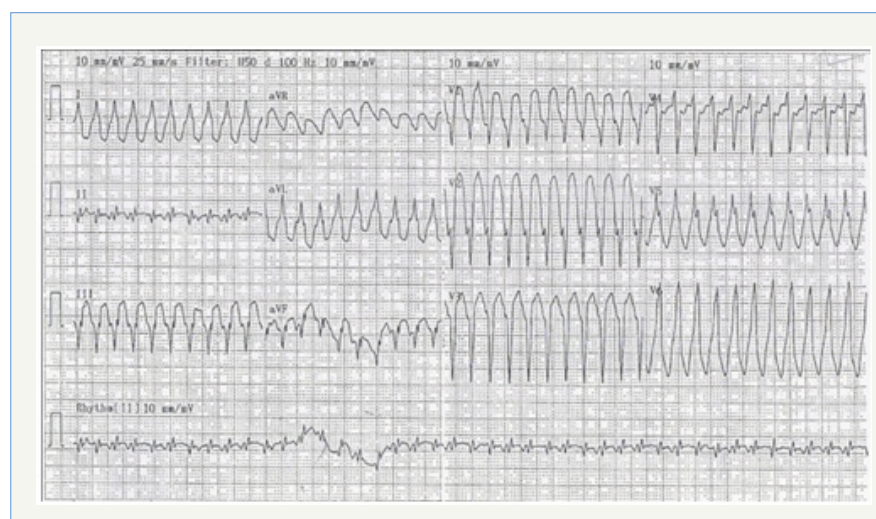

Figure 1: ECG showing wide complex tachycardia on the date of admission.

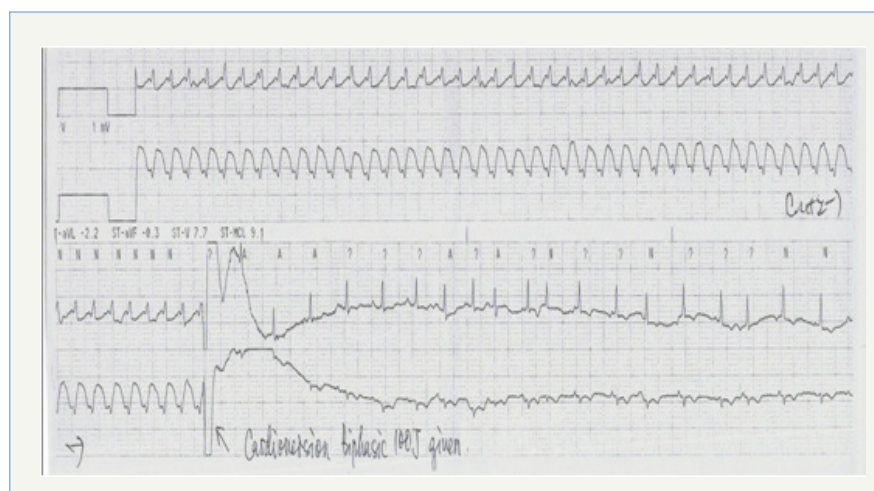

Figure 2: Wide complex tachycardia converted to multifocal a trial tachycardia after cardioversion.

A blood test indicated decreased $\mathrm{TSH}<0.015 \mathrm{miu} / \mathrm{L}$, elevated Free T4 (49.4pmol/L), and FreeT3 (6.8pmol/L); thyroid peroxidase auto anti-bodies were $161 \mathrm{IU} / \mathrm{mL}$ and thymoglobulin auto anti bodies were $>2500 \mathrm{IU} / \mathrm{mL}$. Trop I was $0.079 \mathrm{ug} / \mathrm{L}$. Thyrotoxicosis was suspected. Physical examination revealed that the patient had elevated jugular venous pressure (JVP) and mild peripheral 
edematous; Chest X-ray showed mild cardiomegaly. The patient was prescribed Carbimazole and Lugo iodine. In view of her a trial tachycardia with NSVT, the cardiologist prescribed oral Propranolol 10mg every 8 hours.

The patient's ECG reverted to sinus rhythm an hour after administration of the first dose of Propranolol. However, the patient suddenly developed restlessness, sweating, cyanosis and dyspnea two hours later. BP dropped from $110 / 60 \mathrm{mmHg}$ to $50 / 30 \mathrm{mmHg}$. Fluid challenge was given without effect. Shortly afterwards her cardiac rhythm went into pulse less electrical activity with a junctional rhythm (Figure 3).

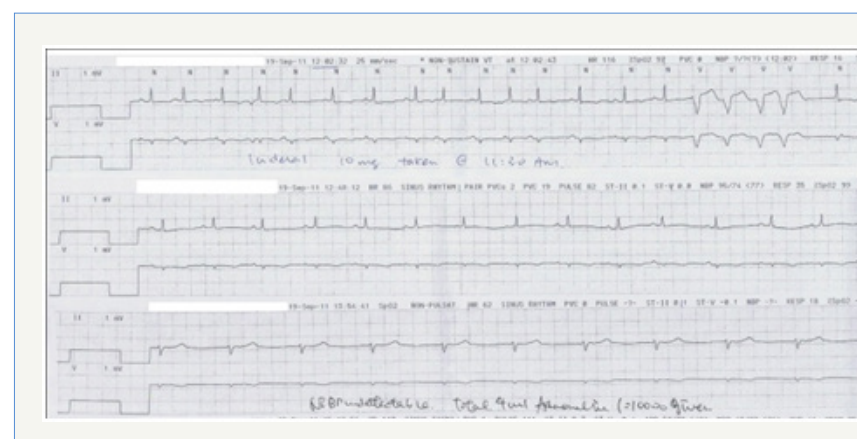

Figure 3: Pulse less electrical activity with junctional rhythm a couple of hours after taking Propranolol.

The patient was incubated and resuscitated for 30 minutes High-dose adrenaline with $100 \mathrm{mcg} / \mathrm{min}$ was required. She developed acute pulmonary edema during resuscitation, which was improved by high-positive end-expiratory pressure (PEEP) ventilation support.

An emergency echocardiogram showed poor LVEF 30\% with mild dilated left ventricular size, severe tricuspid regurgitation without pulmonary hypertension, and mild aortic and mitral regurgitation. Chest radiography showed patchy shadowing over the lungs and right pleural effusion, which suggested fluid overload and/or heart failure. An intra-aortic balloon pump (IABP) was inserted for cardiogenic shock. Her ECG rhythm remained in sinus tachycardia after successful resuscitation. However, a repeated echocardiogram on the next day showed deteriorating cardiac function with LVEF $20 \%$ and she developed mild pulmonary hypertension.

Laboratory results revealed liver and renal failure with markedly elevated ALT, AST and creatinine levels, with metabolic acidosis and hyperkalemia. Continuous renal replacement therapy (CRRT) was started for hyperkalemia and renal failure. Twelve hours of therapeutic hypothermia was commenced due to prolonged cardiac arrest. Pharmacological treatments included Carbimazole, Lugo iodine, hydrocortisone, Vitamin K1, lactulose and empirical antibiotics.

\section{Outcome and Follow up}

Her ECG remained in sinus rhythm throughout. Repeated echocardiogram on day 8 showed improved LVEF of $50 \%$. Her liver and renal function improved afterwards. The patient was weaned off continuous renal replacement therapy, inotropic support and subsequently extubated on day 9.

\section{Discussion}

Thyroid hormones have significants effects on the heart and cardiovascular system through many direct and indirect pathological mechanisms [2]. Increased thyroid hormone activates the renin-angiotensin system and magnifies the sensitivity of beta-adrenergic receptors to catecholamine, which increases left ventricular contractility and heart rate. The activation of the reninangiotensin system also decreases systemic vascular resistance, and increases blood volume [5-7]. The chain of reaction subsequently increases myocardial oxygen consumption, cardiac output and cardiac workload [1].

In thyrotoxicosis, the reduced electrical threshold of a trial depolarization usually leads to rhythm disturbance [7]. A trial fibrillation occurs in $10-15 \%$ of patients with hyperthyroidism [7]. In contrast, it is uncommon to present with paroxysmal supraventricular tachycardia, or wide complex supraventricular tachycardia as demonstrated in this case $[3,8]$. According to Jao, only three cases reported ventricular tachyarrhythmia in relation to thyroid problems [9]. One of the cases developed coarse ventricular fibrillation after palpating the thyroid gland during surgery; another case was a 4-year-old boy who developed monomorphic ventricular tachycardia during febrile convulsion. His ventricular tachycardia was not responsive to anti-arrhythmic drugs but was to anti-thyroid treatment [9].

Longstanding hyperthyroidism exaggerates hypervolemic burden, sinus tachycardia and a trial fibrillation, resulting in rate-related left ventricular dysfunction and heart failure $[3,10]$. Sometimes, right heart strain causes pulmonary artery hypertension which is reflected by neck vein distension and peripheral edema [10]. Clinically, the case presented in this article developed heart failure as shown by the patient's elevated JVP, mild edematous and mild cardiomegaly in chest radiograms. Young individual swithlong-standing hyperthyroidism usually develop 'high-output' heart failure in the absence of any underlying heart disease [3]. Young individuals may have a better increase in cardiac workload to cope with the hemodynamic burden, i.e. the increased preload and total blood volume. This compensation, on the other hand, leads to myocardial hypertrophy [3]. The increased preload and blood volume also elevate ventricular filling pressures, leading to pulmonary and peripheral congestion [3].

The cardiologist prescribed Propranolol for the presented casein view of tachyarrhythmia related to thyrotoxicosis. Betaadrenergic blockers are the conventional therapy for management of such tachyarrhythmia, especially forrate-related heart failure [11]. It slows both the resting and exercise heart rate, thus improving ventricular filling pressure and cardiac output $[9,11]$. This non-selective beta-adrenergic blocker also alleviates beta receptor-mediated symptoms such as anxiety and tremulousness $[1,12]$. A higher than usual dose of beta blockers is suggested to 
enhance beta-adrenergic responsiveness as thyrotoxicosis is associated with an increased number of responsive beta-adreno receptors and plasma clearance $[7,12]$.

However, the case went into cardiovascular collapse after taking propranolol. There was a total of six cases reporting cardiovascular collapses after taking Propranolol in thyrotoxicosis or severe hyperthyroidism from 2000 to 2016. In those cases, four were Chinese reported by Singapore professionals $[3,13]$. The same as the case presented in the article, most of the reported cases developed cardiac arrest 3 to 4 hours after taking Propranolol orally. Even though most of them had certain signs of heart failure or cardiomegaly, Ashikaga reported a case without signs of heart failure which developed frank cardiac failure with four-chamber dilatation and markedly reduced ejection friction after taking oral Propranolol [14]. All six reported cases were found to have decreased ejection fraction to a fatal level, and a certain degree of cardiac impairment such as four-chamber dilatation or severe mitral/tricuspid regurgitation. Half of the reported cases required IABP and one received Extracorporeal Membrane Oxygenation (ECMO) therapy. Half were discharged while another half died subsequently.

Even though Propranolol is conventionally used in the control of tachycardia in patients with thyrotoxicosis, it depresses myocardial contractility, resulting in sharply decreased cardiac output and cardiovascular collapse [3]. Ikram [10] revealed that a beta-adreno receptor blockade has a deleterious effect on cardiac function as the medication reduces the compensatory effect on the autonomic activity in hyperthyroid heart failure [10]. The use of beta blockers should be carefully considered, even avoided, in patients who may have thyrotoxic cardiomyopathy as evidenced by longstanding hyperthyroidism and cardiomegaly in chest radiography $[8,12]$. A lower dose of Propranolol, such as $0.5 \mathrm{mg}$ intravenously should be instituted instead of the full dose in the first instance $[7,14]$.

It is suggested that cardiac ejection fraction and left ventricular size be assessed to guide beta-blocker choices [4]. When left heart failure or low cardiac output is detected, short-acting beta-blockers such as esmolol can be considered [7]. Having an equipotent effect to Propranolol, Esmolol has been used successfully in managing tachyarrhythmia and a trial fibrillation in hyperthyroid patients [2]. Esmolol is a better option than other beta-blockers. Esmolol has a short elimination half-life ( $9 \mathrm{~min}$ ) which facilitates rapid titration and prompt weaning if complications arise [2]. Esmolol should be used under hemodynamic monitoring to monitor its significant adverse effect of hypotension [2].

If a patient is diagnosed with thyrotoxic cardiomyopathy with hemodynamic instability, treatment should focus on acute heart failure, such as emergency intubation with ventilatory support, high-dose inotrope and hemodynamic support, e.g. IABP [11]. A beta-blockade should be avoided.

\section{Learning Points}

When treating tachyarrhythmia in thyroid storm:

A. Propranolol may decrease LVEF to a fatal level and lead to cardiovascular collapse;

B. Propranolol should be used highly cautiously, especially in the case of thyrotoxic cardaic failure;

C. Earlycardiac assessmentis suggested to guide the use of Propranolol;

D. Short-acting beta-blockers such as esmolol could be considered, especially in patients with thyrotoxicosis complicated by left ventricular failure.

\section{References}

1. Toft A, Gaskell K (2009) Treatment options for primary thyroid disease in patients with heart failure. In: Iervasi G, Pingitore A (Eds.), Thyroid and heart failure. Springer-Verlag, Italy, pp. 215-218.

2. MisumiK, Kodera S, Nagura F, Kushida S, Shiojiri T, et al. (2016) Cardiac arrest caused by landiolol in a patient in thyroid crisis. Journal of Cardiology Case 14(2): 62-64.

3. Ngo ASY, Tan DCL (2006) Thyrotoxic heart disease. Resuscitation 70(2): 287-290.

4. Eleftherious G, Butera R, Mantovani L, Bacis G, Manzo L (2011) Myocardial arrest associated with propranolol use in thyroid storm. Reaction weekly (1363): 35.

5. Wang YY, Jiao B, Guo WG, Che HL, Yu, ZB (2010) Excessive thyroxine enhances susceptibility to apoptosis and decreases contractility of cardiomyocytes. Mol Cell Endocrinol 320(1-2): 67-75.

6. Jevalikar G, Gupta P, Bhatia V, Kapoor A, Gambhir S (2011) Paradoxical euthyroid hormone profile in a case of graves' disease with cardiac failure. Int J Pediatr Endocrinol 2011: 8.

7. Reddy V, TahaW, KundumadamS, Khan M (2017) Atrial fibrillation and hyperthyroidism: A literature review. Indian Heart Journal 69(4): 545550 .

8. Ngo ASY, Chew HC (2007) When the storm passes unnoticed-a case series of thyroid storm. Resuscitation 73(3): 485-490.

9. Jao YTFN, Chen Y, Lee WH, Tai FT (2004) Thyroid storm and ventricular tachycardia. South Med J 97(6): 604-607.

10. Ikram H (1977) Haemodynamic effects of beta-adrenergic blockade in hyperthyroid patients with and without heart failure. Br Med J 1(6075): 1505-1507.

11. Soh MC, Croxson M (2008) Fatal thyrotoxic cardiomyopathy in a young man. BMJ 337: a531.

12. Ross DS, Cooper DS, Mulder JE (2017) Beta blockers in the treatment of hyperthyroidism.

13. Ashikaga H, Abreu R, Schneider RF (2000) Propanolol administration in a patient with thyroid storm. Annals of Internal Medicine 132(8): 681682.

14. Fadle BM, Ellahham S, Ringel MD, Lindsay J, Waetofsky L, et al. (2000) Hyperthyroid heart disease. Clin Cardiol 23(6): 402-408. 